\title{
Conflict or congruence? Maternal and infant-centric factors associated with shorter exclusive breastfeeding durations among the Tsimane
}

Keywords: Bolivia; breastfeeding; complementary feeding; infant and young child feeding practices; indigenous health; premastication; low milk supply

\begin{abstract}
Six months of exclusive breastfeeding (EBF) is considered optimal for infant health, though globally most infants begin complementary feeding (CF) earlier-including among populations that practice prolonged breastfeeding. Two frameworks for understanding patterns of early CF emerge in the literature. In the first, maternal and infant needs trade-off, as "maternalcentric" factors-related to time and energy demands, reproductive investment, cultural influences, and structural barriers - favor supplanting breastfeeding with earlier and increased CF. A second framework considers that "infant-centric" factors-related to infant energetic needs-favor CF before six months to supplement breastfeeding.

We apply these two frameworks in examining early CF among the Tsimane-a highfertility, high-mortality, forager-horticulturalist population residing in the Bolivian Amazon. Data were collected from a mixed-longitudinal sample of 161 Tsimane mother-infant pairs from August 2012 - April 2013. Tsimane mothers generally reported introducing CF because of perceived infant needs. However, $\mathrm{CF}$ is introduced with continued intensive breastfeeding, and generally coupled with premastication. Risks of earlier CF relative to the minimum hazard (estimated at 5 births) were elevated for lower and higher parity mothers, but were significantly greater only after 9 births. Seventeen percent of mothers reported introducing CF because of low
\end{abstract}


milk supply. Introducing CF because of low milk was most common from 0-3 months of age and among higher parity mothers, which may reflect physiological constraints. Maternal reproductive trade-offs and perceived infant needs may help explain the low prevalence of EBF to six months among other populations in which breastfeeding is not structurally or culturally constrained.

\section{Introduction}

Six months of exclusive breastfeeding $(\mathrm{EBF})$ reduces infant morbidity and mortality in a wide range of environments (Black et al., 2008; Hop et al., 2000; Kalanda et al., 2006; Kramer and Kakuma, 2002) and is sufficient to support growth under optimal conditions (Dewey, 1998; Dewey et al., 1991). EBF as compared to predominant breastfeeding is associated with longer lactational amenorrhea, while longer durations of breastfeeding reduce maternal risks of reproductive cancers and other non-communicable diseases (Chowdhury et al., 2015; Victora et al., 2016). The onset of several developmental features between four and seven months of age further suggests that EBF for six months is biologically appropriate for our species (Hendricks and Badruddin 1992; Humphrey 2010; Sellen 2009).

Globally, however, only about $40 \%$ of infants are EBF to 6 months (Lutter and Morrow, 2013; Victora et al., 2016). In many populations, the barriers to EBF are rooted in a suite of interrelated factors that have prompted declines in breastfeeding since the $20^{\text {th }}$ century-e.g. lack of structural, familial, and community support for breastfeeding, availability of breast milk substitutes, maternal employment, scheduled infant feedings, and other limitations on maternalinfant interactions (Balogun et al., 2015; Harrell, 1981; Huffman, 1984; King and Ashworth, 1987; Wright, 2001). Risks associated with introduction of complementary feeding (CF) before 
six months of age (hereafter: "early CF") may also be minimized when coupled with low infectious disease exposure and high-quality medical care and complementary foods.

Yet early $\mathrm{CF}$ is also readily evident in the ethnographic record and among contemporary small-scale populations in which breastfeeding is universal, prolonged, and on-demand (Sellen, 2001; Veile et al., 2014). Since EBF in these higher infectious disease risk populations would presumably be feasible for mothers and maximally beneficial to infants, we hypothesize that in such populations (a) EBF may be costly to mothers, or (b) mothers perceive that infants benefit from earlier CF.

Evolutionary researchers have proposed that optimal durations of EBF vary according to the costs and benefits of EBF for mothers and infants (McDade and Worthman, 1998; Tully and Ball, 2013). For example, while EBF may benefit the health of mothers and infants, maternal time devoted to nursing cannot be spent on other fitness-enhancing activities, including wage and household labor or care of existing dependents (Hurtado et al., 1992; Panter-Brick, 1993a). From an evolutionary perspective, EBF also limits opportunities for future reproduction, as high suckling frequency and the metabolic costs of lactation combine to suppress ovulatory function (McNeilly, 1997; Valeggia and Ellison, 2009). In populations in which exogenous mortality is high and extra-somatic capital is not readily accrued or transmitted, surplus maternal energy tends to favor higher fertility over investments in health, longevity, or improving conditions of individual offspring (Gibson and Mace, 2006; McAllister et al., 2012; Strassmann and Gillespie, 2002). Under such "maternal-centric" circumstances affecting residual fertility, younger, lower parity mothers may use early $\mathrm{CF}$ as a subconscious strategy to reduce lactational costs, leading to earlier resumption of postpartum ovulation and subsequent pregnancy. 
Alternatively and despite additional risks of pathogen exposure, early CF may promote infant growth and immune maturation, or compensate for insufficient milk quantity or quality. Six months of EBF may not universally meet the needs of all infants given variation in birth size, growth velocity, milk volume, iron and other available nutrients in milk (Fewtrell et al., 2007; Kramer and Kakuma, 2002; Nielsen et al., 2011; Reilly and Wells, 2005; Waterlow, 1981). EBF infants are also not immune from infection, and infants who experience repeated energy losses from pathogen exposure may require supplementation (Waterlow, 1981; Wilson et al., 2006).Turkana, Aka, and Ngandu mothers have reported the belief that infants should be supplemented shortly after birth to buffer against disease risks (Gray, 1998; Meehan and Roulette, 2013). More recent evidence suggests that introducing small amounts of common allergenic foods between 4-6 months may promote immunotolerance (Koplin et al., 2010; Prescott et al., 2008; Wennergren, 2009). Finally, perceived low milk supply is often emblematic of modernized infant care practices that interrupt the positive feedback loop between suckling frequency and milk production (e.g. scheduled nursing bouts, bottle feeding, decreased maternalinfant contact) (Greiner et al., 1981; Gussler and Briesemeister, 1980). However, in populations in which such practices are rare, low milk supply is still reported, often in connection with perceived infant satiety, maternal dietary sufficiency, and labor conflicts (Levine, 1988; Moffat, 2002).

We examine evidence of both maternal- and infant-centric factors influencing $\mathrm{CF}$ practices among the Tsimane, a natural-fertility forager-horticulturalist population residing in the Bolivian Amazon. Tsimane reproductive investment appears directed towards higher fertility despite potential costs to offspring: contraceptive use is rare while high fertility is socially and economically desirable; higher parity-for-age is associated with earlier weaning; child growth 
begins to falter around the time of weaning; and shorter interbirth intervals are associated with higher rates of infant mortality (Gurven et al., 2012; McAllister et al., 2012). The Tsimane are well-nourished and relatively unburdened by non-communicable diseases, including obesity, diabetes, and reproductive cancers (Gurven et al., 2013). We propose that Tsimane mothers are therefore unlikely to consciously vary EBF durations to benefit their own health, but maternalcentric factors, indicative of surplus energy budgets or greater reproductive potential, may be associated with shorter EBF durations.

Conversely, there are few structural or cultural constraints impeding longer breastfeeding durations. Mothers give birth at home, generally begin breastfeeding shortly after birth, and do not ritually discard colostrum (Veile et al., 2014). Though CF is introduced on average at four months, infants are more likely to be observed breastfeeding than they are CF up to 13 months of age (Veile et al., 2014), which suggests early CF may not supplant breastfeeding. Tsimane mothers carry infants in slings throughout the day and "breastsleep" (McKenna and Gettler, 2016) at night. Mothers are rarely absent from infants; subsistence activities are largely domestic (Gurven et al., 2009) and families generally travel together on longer excursions (Ellis, 1996; Miner et al., 2014). As such, early CF may be motivated by infant-centric factors.

The research presented in this paper is organized into three sections. For comparative purposes, we first assess Tsimane breastfeeding and CF practices according to population indicators of age-appropriate infant and young child feeding practices (World Health Organization, 2007). We complement this with ethnographic description of CF practices, and examine Tsimane mothers' reported reasons for introducing CF. Lastly, we evaluate maternal and infant factors in predicting age of CF introduction as reported in ethnographic interviews and in a statistical model. 


\section{Methods}

\section{Study population and data collection}

The Tsimane are an indigenous forager-horticulturalist population residing in the Beni Department of Bolivia. The population is estimated at 15,000, distributed over 90 villages of varying size $(\sim 80-500)$, ecology (riverine floodplain, terra firme forest), and access to market towns (Miner et al., 2014; Reyes-García et al., 2014). Infant mortality rates vary between 10$20 \%$ (Gurven et al., 2007; Gurven, 2012) and the total fertility rate is 9.1 births (McAllister et al., 2012). Parasitism is endemic (Blackwell et al., 2013; Martin et al., 2013), while access to medical care and family planning services is limited (McAllister et al., 2012).

A mixed-longitudinal study was conducted across nine Tsimane villages from September 2012 - April 2013. The villages vary with respect to river access and distance to the market town of San Borja (pop. 24,000) (Supplementary Table 1[INSERT LINK TO ONLINE SUPPLEMENTARY FILE]). All families with children aged 0-35 months were asked to participate. One family declined, and interviews were not successfully coordinated with another 13 families who agreed to participate (random with respect to village and infant age). The final sample included 161 families from 150 households, representing 92\% of all eligible families present.

Ethnographic interviews, dietary recall, and anthropometric measures were collected during in-home visits. To examine longitudinal changes in feeding practices, a follow-up study was conducted with a subset of 48 infants from six villages who were less than 12 months old at initial interviews. Monthly dietary recalls and anthropometric measures were collected from these subjects over the next 4-6 months. The number of follow-up visits per subject varied due to 
date of initial interview and family absence during visits to communities (2-6 visits per subject, mean $\pm \mathrm{SD}=3.2 \pm 1.4$ ). Descriptive statistics for subjects by study group are given in Table 1 .

At every visit, 24-hour dietary recalls were first conducted to establish infant feeding status. Following WHO (2007) guidelines (Supplementary Table 2 [INSERT LINK TO ONLINE SUPPLEMENTARY FILE), EBF was defined as consumption of breast milk only, and CF as breastfeeding with any other solids or liquids (including water and formula). Weaning was defined as the full cessation of breastfeeding. At initial interviews, 30 infants were EBF, 106 had already begun $\mathrm{CF}$, and 25 were already weaned. Among the infants sampled prospectively, 20 were EBF and 24 were CF when first interviewed. Over the course of the prospective study, 15 of these EBF infants transitioned to $\mathrm{CF}$, and all infants were still breastfeeding at the end of the study.

Interviews were conducted in the Tsimane language by a trained assistant, with MM or GG present. MM conducted interviews in Spanish with ten mothers of mixed ethnicity who spoke Spanish as their primary language. The initial interview consisted of short-answer questions about the focal child's birth, breastfeeding, complementary feeding, and premastication practices. Mothers of non-EBF infants were asked what the first foods and liquids they gave to their infants were, the age at which they gave them, and why they started CF. The earlier age of first food or liquid was recorded as age at CF. For EBF infants in the follow-up study, age at CF was recorded from subsequent dietary recalls. Maternal height was measured to the nearest $0.1 \mathrm{~cm}$ using a portable stadiometer (Seca 217). 
In statistical analysis, we consider three proxies of maternal condition expected to favor future over current reproductive investment, and therefore earlier CF: greater maternal height, lower parity, and a greater number of household females over the age of 7 . Though maternal height is an indication of health and nutrition during childhood, these conditions may reflect access to family support and other resources that persist into adulthood. As an indicator of relatively improved condition and access to resources that can be invested in higher fertility, we predict that greater height will be associated with earlier CF. Younger mothers at lower parity are also expected to introduce CF earlier, as these mothers are presumed to subconsciously favor future reproductive investment. Finally, Tsimane females over the age of 7 (who include maternal offspring, siblings, and other relatives) make significant contributions to child care (Winking et al., 2009). Mothers may divert more infant care to these allomothers, and thus begin CF earlier.

Infant-centric factors considered included sex and birth season. Milk composition in humans and other animals has been observed to vary by infant sex (Fujita et al., 2012; Hinde, 2009; Hinde et al., 2014; Powe et al., 2010; but see Quinn, 2013), which may differently affect EBF durations. Rainy season births among the Tsimane have been associated with lower maternal BMI and greater morbidity risks for infants (Gurven, 2012), which may negatively influence maternal lactation or infant birth size.

We also considered the influence of village location, as a previous study showed that EBF durations in remote Tsimane villages were shorter than in villages closer to market (Veile et al., 2014). Perceptions of low milk and infant size at CF introduction are evaluated in limited analyses, as these were reported only in conjunction with reasons for CF introduction in initial interviews. In our mixed-longitudinal design, infant age relative to current feeding status and age 
at $\mathrm{CF}$ introduction varied widely across subjects. As such, age at $\mathrm{CF}$ was not evaluated in terms of time-varying factors such as maternal weight, \% body fat, BMI, infant weight, length, or illness bouts.

Ethical approval

All study protocols were approved by the University of California Santa Barbara Institutional Research Board Human Subjects. Permission to conduct research was granted to the Tsimane Life History Project (THLHP) and affiliates. The THLHP maintains formal agreements with the local municipal government of San Borja and the Tsimane governing body (Gran Consejo Tsimane'). Research permission was also granted independently to MM by the Estación Biológica de Beni.

The purpose of the study was explained to each of the study villages in community meetings held prior to beginning data collection. Subjects gave informed consent orally before each interview and follow-up visit. Subjects were compensated for their time with small care packages that included household goods (e.g. yarn, thread, combs) and over-the-counter medicines (e.g. paracetamol, salve).

\section{Statistical Analysis}

All statistical analyses were performed using R (ver. 3.2.1). EBF and breastfeeding durations were estimated using multiple methods. First, the proportion of infants EBF at 0-5 months was estimated following WHO (2007) guidelines (Supplementary Table 2), using current feeding status of infants aged 0-5 months only. Second, mean \pm SE age of CF introduction for all 
children surveyed was estimated by Kaplan-Meier survival analysis, with EBF infants censored at age of interview. Ages of CF introduction reported during initial interviews and in follow up dietary recalls did not significantly differ (mean $\pm \mathrm{SE}=130 \pm 5$ vs. $135 \pm 8$ days, log rank test $p$ $=0.72$ ), and were combined in subsequent analyses. All weaning ages were reported from maternal recall in initial interviews. Mean \pm SE age at weaning was also estimated by KaplanMeier survival analysis for all children aged 12-35 months (no children were weaned before one year of age). For all non-EBF children, mean \pm SD age at $C F$ introduction and weaning as reported in initial interviews were calculated by village, infant sex, age group, and maternal parity group.

Age of CF in association with perceptions of infant growth and milk supply at time of CF introduction was examined by chi-square. Variance in premastication was assessed in a generalized mixed-effects model using the lme4 package (Bates et al., 2014). The model included maternal ID as a random effect and infant age and meat and fish consumption the day prior as controls. We included maternal age, maternal Spanish fluency ("none” vs. “conversational to fluent") and village location (remote vs. near market) as proxies of a more traditional lifestyle, predicting that younger and more acculturated mothers would be less likely to report premastication.

A Cox proportional hazards model was used to assess variation in EBF duration in association with maternal- and infant-centric factors. We first constructed a baseline Cox model that included the five maternal and infant factors discussed above, along with village location, and the time elapse since the reported age at $\mathrm{CF}$ introduction (to control for recall bias). A penalized spline term for parity was used upon further examination of the data. We determined the best fit Cox model using backwards stepwise selection with AICc, and evaluated the model 
for proportional hazards assumptions, influential observations, and nonlinearity of continuous variables. The final model was fit using the smoothHR package (Meira-Machado et al., 2013), which computes optimal degrees of freedom as specified by AICc, and estimates log hazard ratios and corresponding confidence intervals for nonlinear continuous variables.

The relationship between perceived low milk and age at CF introduction was assessed via linear regression. This analysis was limited to responses from non-EBF mothers in initial interviews $(\mathrm{n}=127)$. The model included the same covariates from the final Cox model, substituting a parity group category in place of the non-linear spline, and adding a binary term for whether or not CF was introduced owing to perceived low milk supply. The model was checked for interactions among covariates and assumptions of normality.

\section{Results}

\section{Tsimane infant feeding practices}

According to population estimates based on 24-hour dietary recall and WHO specified age groups (Supplementary Table 2), all Tsimane infants have been breastfed. Only 53\% of mothers breastfeed within one hour of birth (Table 2), though these times are approximate since Tsimane women do not typically wear watches. All infants are estimated to begin CF by 6-8 months, and all.children are estimated to breastfeed for at least one year, with $87 \%$ still breastfeeding at two years of age (Table 2).

As estimated by Kaplan-Meier survival analysis $(n=161, n$ events $=146)$, mean \pm SE time to CF introduction was $132.6 \pm 4.9$ days (approximately 4.4 months) and mean age at weaning $(\mathrm{n}=100$, events $=21)$ was $824.1 \pm 36.5$ days (approximately 27 months). Although according to WHO indicators $64 \%$ of infants are estimated to be EBF at 0-5 months (Table 2), 
only $25 \%$ of children with known age at CF (38/146) reportedly began CF at the recommended ages of 6-8 months $-75 \%$ began $\mathrm{CF}$ at 0-5 months. Among non-EBF children at initial interviews $(n=130)$, reported mean \pm SD age of $C F$ was $3.8 \pm 2.0$ months, with $41 \%$ reporting $\mathrm{CF}$ before four months of age; mean \pm SD age at weaning was $19.1 \pm 5.9$ months, with only $13 \%$ of children weaned before two years of age. (Supplementary Table 3 [INSERT LINK TO ONLINE SUPPLEMENTARY FILE HERE).

Food costs and preparation time for the most common Tsimane complementary foods appear to be minimal. Mothers do not prepare special foods for infants, though the diet is generally soft and bland, and conducive to infant consumption. Most meals are "jo'na", referring to any rice, plantain, or pasta-based stew mixed with meat or fish. Family members generally eat seated on the ground, consuming individual bowls and additional helpings as desired. Mothers are primarily responsible for feeding infants and young children, though other family members may share snacks and drinks. Before age one, infants sit in their mothers' laps and are spoon- or finger-fed from their mothers' plates. By age two, most children are eating from their own plates, and by age three may be serving themselves liquids, meals, and snacks.

Introduction of liquids and solids is not rigidly imposed. Among non-EBF mothers $(\mathrm{n}=$ 131), $38 \%$ introduced liquids first, $23 \%$ introduced solids first, and 39\% introduced both at the same age. However, solid foods in early months frequently referred to 'chinsi', the clear broth portion of jo'na. Chicha (a fermented beverage made from manioc, corn, or plantain), water, and juice were the most common first liquids introduced. Liquids were served in erepas (hollowed out Crescentia cujete gourds), plastic cups, and occasionally bottles. Though $11 \%$ of infants aged 0-23 months in initial dietary recalls were reportedly bottle-fed the day prior with non-maternal milk liquids (Table 2), none were given liquids exclusively by bottle. Infants in near market 
villages were no more likely to have been bottle-fed than infants in more remote villages (14\% vs. $7 \%$, chi-square $=0.95, p=0.33)$.

Powder infant formula (a $\sim 400 \mathrm{~g}$ cereal box) is available in market kiosks, but costs about 40 bs ( \$US 6), equivalent to more than a half-day’s wage labor. Through a government program, infants at least 6 months old were eligible to receive one 30 -serving bag of formula per month. These could be obtained in San Borja and were sporadically given out by visiting health promoters. When received, formula was given to infants but also consumed in powder form by other family members.

Tsimane mothers frequently premasticate foods deemed too hot, too dry, too tough, or a choking hazard (most commonly jo'na, roasted plantains, smoked or dried meats, and fish). Mothers reported occasionally orally transferring liquids mouth-to-mouth, generally when they were traveling and didn't have a drinking vessel handy, or when they wanted infants to drink “a lot" (perhaps in cases of illness or dehydration). In initial interviews, $84 \%$ and $51 \%$ of non-EBF mothers $(n=132)$, respectively, reported having ever premasticated solids or orally transferring liquids. Premastication and orally transferring liquids were reported in 54\% and 9\%, respectively, of all 24-hour dietary recalls, declining in frequency by infant age (Figure 1). In a mixed-effects logistic regression adjusting for infant age, the likelihood of premastication the day prior was greater with consumption of fish, but did not vary with consumption of meat, village region, maternal age, or maternal Spanish fluency (Supplementary Table 4 [INSERT LINK TO ONLINE SUPPLEMENTARY FILE HERE).

Tsimane mothers' self-reported reasons for introducing CF 
In open-ended questions, $88 \%$ of mothers (121/137) cited specific reasons for introducing $\mathrm{CF}$, with the remaining $12 \%$ reporting "no reason/just because". Responses were grouped into nine categories (Figure 2). Overall, 64\% of mothers followed infant cues in introducing $\mathrm{CF}-$ either perceiving that their infants were hungry, big, or developmentally ready for food, or sick, small and in need of supplementation. Higher proportions of mothers who introduced CF at recommended ages (6-7 months) vs. earlier (0-5 months) reported doing so because infants were hungry, grabbing at food, big/growing well, or "just because" (Supplementary Table 5 [INSERT LINK TO ONLINE SUPPLEMENTARY FILE HERE). All mothers who reported that their infants were small or sick, or that they themselves were sick or had no/low milk, introduced CF before 6 months of age. Sixty-five percent of mothers who reported no/low milk (15/23) introduced CF within the first month of life.

Responses that infants were crying or hungry may represent the same perception. Mothers used several terms to describe infant crying (shavnaqui, shavñye, tari, váti, vara'shaqui), which translate to wailing, crying with tears, and loud or incessant crying. Across 188 hours of daytime focal follows, infants aged 0-16 months were observed crying in less than $1 \%$ of 5-minute observational intervals (Martin, 2015). The onset of persistent crying may therefore be an honest signal of energetic needs or interest in food.

Mothers reporting that their infants were small at birth relative to other Tsimane newborns were no more likely to introduce CF before 4 months of age than mothers who perceived their infants as the same size or larger than other newborns ( $42 \%$ vs $38 \%$, chi-square $=$ $0.05, p=0.814)$. However, mothers who reported their infants as small at the time of $C F$ introduction were more likely to have introduced $\mathrm{CF}$ at $0-3$ months as compared to mothers who 
reported their infants as similar to or larger than other same-aged infants (59\% vs. 31\%, chisquare $=8.05, p=0.004)$.

Seventeen percent of mothers cited low milk supply as a reason for introducing CF. In their own words they had "no breast milk" (itŝij tashin) or were "dry" (chañej). These mothers were no more likely to initiate breastfeeding 24 hours after birth than were mothers who introduced CF for other reasons (chi-square $=0.71, p=0.39$ ). However, $95 \%$ of these mothers introduced $\mathrm{CF}$ at 0-3 months of age, compared to just $32 \%$ of mothers who reported introducing $\mathrm{CF}$ for other reasons (chi-square $=30.41, \mathrm{p}<0.001)$. These mothers were also more likely to report their infants as small for their age at the time of CF introduction, as compared to mothers who introduced CF for other reasons $(71 \%$ vs. $28 \%$, chi-square $=15.24, \mathrm{p}<0.001)$. We did not ascertain whether mothers blamed perceived low milk for small infant size or if mothers gauged low milk from perceived poor infant growth or other cues. However, some mothers reported taking active steps to increase milk supply, such as drinking more liquids and massaging breasts with salt or warm papaya skins, and others reported that problems self-resolved and subsequently began EBF. All mothers who reported low milk continued breastfeeding.

In a separate line of questioning, 40\% (49/125) of mothers reported receiving advice to introduce $\mathrm{CF}$. Of these, $46 \%$ were advised to begin $\mathrm{CF}$ by their own mothers or mothers-in-law, $31 \%$ by other kin, $8 \%$ by other Tsimane, and $14 \%$ by a napo (a non-Tsimane Bolivian national). Advice to begin CF generally echoed the same reasons cited by mothers-because of crying or hunger (59\%), so that infants would grow well and be healthy (13\%), because the infant was small or sick (11\%), or because the mother had no milk (9\%). Primiparous mothers did not report receiving advice any more frequently than did multiparous mothers $(40 \%$ vs. $43 \%$, chi-square $=$ $0.0005, p=0.98)$. 
In initial interviews $41 \%$ of mothers (60/146, including those still EBF) reported ever getting professional advice from a doctor, nurse, or other health care worker about when to feed their infants. However, only 53\% of those mothers were told to introduce CF at 6 months: $25 \%$ were told to begin later than 6 months, 17\% earlier, and the remaining 5\% did not recall any specific age. Of those who received professional advice, 69\% (37/54) introduced CF earlier than they had been recommended.

\section{What predicts timing of $C F$ introduction?}

The best fit Cox model for time to CF included maternal height, parity, and the time elapse since CF introduction (Table 3). Contrary to our prediction, taller mothers were more likely to initiate CF later: each additional centimeter of height was associated with a 5\% lower $\mathrm{CF}$ risk. The penalized spline for parity resulted in a U-shaped hazard curve with the minimum hazard (i.e. longest EBF duration) at five births, and women at lower and higher parity more likely to introduce CF earlier (Figure 3). Log hazard ratios of CF were not significantly greater than the minimum at 1-3 births -including for primiparous mothers $(\mathrm{LnHR}=0.37,95 \% \mathrm{CI}=-$ $0.07-0.81)$-but were significantly greater after nine births $(\operatorname{LnHR}=0.45,95 \% \mathrm{CI}=0.01$ 0.89). The number of household females over age seven, village region, infant sex, and birth season were eliminated during model selection based on AICc. A model substituting a nonlinear term for maternal age instead of parity resulted in a poorer fit, though the resulting hazard curve was similar to that observed for parity (Supplementary Figure 1 [INSERT LINK TO ONLINE SUPPLEMENTARY FILE HERE).

In a linear regression adjusting for covariates, mothers who reported introducing $\mathrm{CF}$ because of perceived low milk began CF on average 3.4 months earlier than those who 
introduced CF because of other reasons (Table 4). As in the survival analysis, greater maternal height was associated with later CF introduction, and primiparity with earlier CF introduction. The association between higher parity and earlier CF introduction appears mediated by perceived low milk, as is evident when low milk is excluded or included in the model (Table 4). Fortyseven percent of high parity (>8 births) mothers (8/17) reported introducing CF because of perceived low milk, as compared to only $13 \%$ of mothers with $2-8$ births (13/84) and $13 \%$ of primiparous mothers (3/20). There were no significant interactions between parity group and low milk or height.

\section{Discussion}

Tsimane mothers' self-reported reasons for introducing CF largely reflect concern for infant needs, with very early CF (0-3 months) notably related to perceived low milk supply and poor infant growth. In general, Tsimane mothers' motivations for early CF are consistent with those of mothers in other intensive breastfeeding populations, who view early supplementation as a practice that benefits, not risks, infant health (Gray, 1998; Meehan and Roulette, 2013). Mothers who were aware of feeding recommendations largely ignored them and introduced CF earlier.

No mothers cited household, labor demands, or lack of support as reasons for introducing CF. Our prediction that factors indicative of maternal reproductive trade-offs would be associated with earlier CF was not strongly supported. Greater maternal height predicted later CF introduction, suggesting mothers in better condition or with better access to resources invest more in current offspring (via more intensive breastfeeding) than in future reproduction (via reduced lactational costs). Additional research is needed to assess if maternal height is correlated 
with other measures of current maternal condition—e.g. energy balance, health, or milk volume and nutritional content — which would suggest that they can better sustain the energetic costs of longer EBF.

As predicted, lower parity was associated with earlier CF, but so was very high parity. This latter finding is inconsistent with an evolutionary framework that predicts reproductive efforts will increase as opportunities for future reproduction decrease (i.e. the terminal investment hypothesis) (Williams, 1966). We propose that different mechanisms may influence EBF duration across the reproductive lifespan. At earlier ages and low parity, maternal inexperience and/or reproductive trade-offs may favor earlier $\mathrm{CF}$, whereas lactational sufficiency may be constrained at advanced ages and higher parity. Higher parity Tsimane mothers more frequently reported introducing CF because of low milk, and indeed the association between high parity and early CF was no longer significant when controlling for perceived low milk (Table 4).

To our knowledge, this is the first study to explicitly link perceived low milk supply with high parity. Though behavioral mechanisms influencing milk supply among this demographic cannot be ruled out, several physiologically mediated mechanisms related to advanced age or parity could negatively impact milk synthesis. In the U.S., advanced maternal age has been linked to delayed onset of lactogenesis, which may result from age-associated increases in glucose intolerance and inflammation (Nommsen-Rivers et al., 2012). Breast milk from Gambian mothers with 10 or more births contained less endogenously synthesized fatty acids than milk from lower parity mothers (Prentice et al., 1989). Fatty acid composition could affect infant satiety or growth and be interpreted as insufficient milk supply. Finally, calcium stores become increasingly depleted with age and successive bouts of lactation (Horst et al., 1997; Nordin, 1997). Tsimane women lose relatively more bone mineral density with age as compared to 
American women, and bone mineral density is further reduced by relatively high parity and short interbirth intervals (Stieglitz et al., 2015). With sustained calcium secretion at high parity, homeostatic mechanisms may downregulate milk supply to conserve essential maternal function. This hypothesis may be tested in future research examining Tsimane milk composition.

In an earlier study of Tsimane infant feeding practices, Veile et al. (2014) found that EBF durations were shorter in remote forest villages as compared to near market villages. In this study, village region was eliminated as a predictive factor of CF during model selection. However, our sample did not include forest villages, while ages of CF between riverine and near market villages in the earlier study did not significantly differ. Of note, Veile et al. (2014) could not determine if longer EBF durations in near market villages resulted from relatively improved conditions, or whether public health messages influenced maternal behavior or biased maternal recall. Our results support the first interpretation, as greater maternal height and perceptions of low milk supply and poor infant growth were associated with earlier CF, but knowledge about feeding recommendations had little influence on CF decisions. At the same time, we observed marked differences in age at CF between individual villages (Supplementary Table 3), which may reflect unexplored influences of family and neighbors on individual feeding decisions.

The age at weaning estimated by survival analysis in this study (27 months) is substantially later than mean ages ( $\sim 19$ months) reported from recall of already weaned infants both in this study (Supplementary Table 3) and in Veile et al. (2014). The age of CF estimated by survival analysis (4.4 months) was slightly higher than ages reported strictly from recall of nonEBF infants both here and in Veile et al. (3.8 and 4.1 months, respectively). CF and weaning ages calculated solely from recall appear biased by exclusion of infants that are still EBF and 
breastfeeding, respectively (i.e. right-censored). For small populations, mixed use of recall with current feeding status may best approximate typical ages of CF introduction and weaning.

The risks of earlier weaning and increased infectious disease exposure associated with early $\mathrm{CF}$ - as well as maternal perceptions of these risks - may vary across populations given maternal motivations, CF and breastfeeding practices, and local ecologies (Moffat, 2001). In many small-scale societies, kin support, divisions of labor, and other cultural practices are integral in maintaining intensive and prolonged breastfeeding (Meehan and Roulette, 2013; Panter-Brick, 1991; Piperata, 2009). In populations in which early CF supplements without supplanting breastfeeding, EBF duration may be a poor indicator of breastfeeding intensity. Indeed, across lower and middle income countries, rates of EBF at 0-5 months are only weakly correlated with rates of breastfeeding at one year or more (Victora et al., 2016), with 60-90\% of infants in these populations still breastfeeding at 12-15 months, despite low rates of EBF at 0-5 months (Lutter and Morrow, 2013). In other environments, the increased energy provided by early CF may paradoxically offset any additional risks posed by contaminated foods (Wilson et al., 2006), while premastication may further minimize infectious exposure risks (Pelto et al., 2010). Finally, any perceived immediate benefits of early CF (e.g. weight gain, placated crying) may outweigh or be easier to discern than long-term risks, including later growth faltering, earlier weaning, and maternal health or reproductive consequences.

However, we caution that additional research is needed to fully ascertain the real or perceived costs and benefits of varying EBF durations for Tsimane mothers and infants. Our study is limited by the mixed-longitudinal design and reliance on maternal recall, which does not allow us to corroborate maternal perceptions of infant size, growth, health, or milk supply at the time of CF introduction. Subtle differences in CF practices and maternal work patterns may still 
influence EBF duration and weaning, but must be assessed by direct observation of breastfeeding and maternal time allocation (Panter-Brick, 1993b). Tsimane infant outcomes may vary with availability and quality of complementary foods, while early CF—even if motivated by infant needs - may still increase pathogen exposure or reduce nursing intensity enough to lead to earlier weaning. Future research with the Tsimane will consider the timing of CF introduction in association with subsequent breastfeeding behaviors, maternal energy balance, and infant growth and health outcomes.

\section{Conclusion}

International health organizations recommend EBF for six months as a universal standard of optimal infant feeding practices. Globally, however, most infants are not EBF for six months. Epidemiological and evolutionary research often frames early $\mathrm{CF}$ as suboptimal for infants-a consequence of structural barriers to breastfeeding, maternal energetic and time constraints, or reproductive trade-offs. However, mothers in diverse settings frequently cite concerns with infant growth, health, or milk supply as reasons for introducing CF before six months. While both maternal- and infant-centric factors likely combine to influence individual EBF durations, the contextual environment in which breastfeeding occurs may be important in predicting whether or not shorter EBF durations reflect maternal-infant conflict or congruence. Such a framework may also be useful in predicting risks of early $\mathrm{CF}$ in specific environments, and developing locally salient interventions to improve infant outcomes.

Though there are few if any structural barriers constraining breastfeeding among the Tsimane, we proposed that reproductive trade-offs may still favor shorter EBF durations in this well-nourished, high fertility population. Instead our results suggest that motivations for early CF 
are primarily infant-centric - reflecting differences in perceived infant needs that may reflect individual variation in infant growth or lactational sufficiency. In partial support of our predictions, we found a novel non-linear association between parity and earlier $\mathrm{CF}$, and suggest that different behavioral and/or physiological mechanisms may constrain EBF among primiparous and high parity Tsimane mothers. Additional research on maternal and infant outcomes associated with EBF durations may better illuminate the role of reproductive trade-offs in influencing the causes and consequences of early CF in this population.

\section{References}

Balogun, O.O., Dagvadorj, A., Anigo, K.M., Ota, E., Sasaki, S., 2015. Factors influencing breastfeeding exclusivity during the first 6 months of life in developing countries: a quantitative and qualitative systematic review. Matern. Child Nutr. 1-19. doi: $10.1111 / \mathrm{mcn} .12180$

Bates, D.M., Mächler, M., Bolker, B., Walker, S., 2014. Fitting Linear Mixed-Effects Models using lme4. J. Stat. Softw. 1406, 1-51.

Black, R.E., Allen, L.H., Bhutta, Z.A., Caulfield, L.E., de Onis, M., Ezzati, M., Mathers, C., Rivera, J., 2008. Maternal and child undernutrition: global and regional exposures and health consequences. Lancet 371, 243-60. doi:10.1016/S0140-6736(07)61690-0

Blackwell, A.D., Martin, M., Kaplan, H., Gurven, M., B, P.R.S., 2013. Antagonism between two intestinal parasites in humans: the importance of co-infection for infection risk and recovery dynamics. Proc. R. Soc. Lond. B 280, 20131671.

Chowdhury, R., Sinha, B., Sankar, M.J., Taneja, S., Bhandari, N., Rollins, N., Bahl, R., 
Martines, J., 2015. Breastfeeding and maternal health outcomes: a systematic review and meta-analysis. Acta Paediatr. 104, 96-113. doi:10.1111/apa.13102

Dewey, K.G., 1998. Cross-cultural patterns of growth and nutritional status of breast-fed infants. Am. J. Clin. Nutr. 67, 10-17.

Dewey, K.G., Heinig, M., Nommsen, L., Lönnerdal, B., 1991. Adequacy of energy intake among breast-fed infants in the DARLING study: relationships to growth velocity, morbidity, and activity levels. J. Pediatr. 119, 538-47.

Ellis, R., 1996. A taste for movement: an exploration of social ethnics of the Tsimanes of Lowland Bolivia. St. Andrews University.

Fewtrell, M.S., Morgan, J.B., Duggan, C., Gunnlaugsson, G., Hibberd, P.L., Lucas, A., Kleinman, R.E., 2007. Optimal duration of exclusive breastfeeding: what is the evidence to support current recommendations? Am. J. Clin. Nutr. 85, 635S-638S.

Fujita, M., Roth, E., Lo, Y.-J., Hurst, C., Vollner, J., Kendell, A., 2012. In poor families, mothers' milk is richer for daughters than sons: a test of Trivers-Willard hypothesis in agropastoral settlements in Northern Kenya. Am. J. Phys. Anthropol. 149, 52-9. doi:10.1002/ajpa.22092

Gibson, M.A., Mace, R., 2006. An energy-saving development initiative increases birth rate and childhood malnutrition in Rural Ethiopia. PLoS Med. 3, e87.

Gray, S., 1998. Butterfat Feeding in Early Infancy in African Populations : New Hypotheses. Am. J. Hum. Biol. 178, 163-178. doi:10.1002/(SICI)1520-6300(1998)10:2<163::AIDAJHB3>3.3.CO;2-I

Greiner, T., Van Esterik, P., Latham, M.C., 1981. The insufficient milk syndrome: An alternative explanation. Med. Anthropol. 5, 233-247. 
Gurven, M., 2012. Infant and fetal mortality among a high fertility and mortality population in the Bolivian Amazon. Soc. Sci. Med. 75, 2493-2502.

Gurven, M., Jaeggi, A. V, Kaplan, H., Cummings, D., 2013. Physical activity and modernization among Bolivian Amerindians. PLoS One 8, e55679. doi:10.1371/journal.pone.0055679

Gurven, M., Kaplan, H., Supa, A.Z., 2007. Mortality experience of Tsimane amerindians of Bolivia: Regional variation and temporal trends. Am. J. Hum. Biol. 19, 376-398.

Gurven, M., Stieglitz, J., Hooper, P.L., Gomes, C., Kaplan, H., 2012. From the womb to the tomb: the role of transfers in shaping the evolved human life history. Exp. Gerontol. 47, 807-13. doi:10.1016/j.exger.2012.05.006

Gurven, M., Winking, J., Kaplan, H., von Rueden, C., McAllister, L., 2009. A bioeconomic approach to marriage and the sexual division of labor., Human nature (Hawthorne, N.Y.). doi:10.1007/s12110-009-9062-8

Gussler, J.D., Briesemeister, L.H., 1980. The insufficient milk syndrome: A biocultural explanation. Med. Anthropol. 4, 145-174. doi:10.1080/01459740.1980.9965867

Harrell, B.B., 1981. Lactation and Menstruation in Cultural Perspective. Am. Anthropol. 83, 796-823.

Hendricks, K.M., Badruddin, S.H., 1992. Weaning recommendations: the scientific basis. Nutr. Rev. 50, 125-33.

Hinde, K., 2009. Richer milk for sons but more milk for daughters: Sex-biased investment during lactation varies with maternal life history in rhesus macaques. Am. J. Hum. Biol. 21, 512-9. doi:10.1002/ajhb.20917

Hinde, K., Carpenter, A.J., Clay, J.S., Bradford, B.J., 2014. Holsteins favor heifers, not bulls: biased milk production programmed during pregnancy as a function of fetal sex. PLoS One 
9, e86169. doi:10.1371/journal.pone.0086169

Hop, L.T., Gross, R., Giay, T., Sastroamidjojo, S., Schultink, W., Lang, N.T., 2000. Premature complementary feeding is associated with poorer growth of Vietnamese children. J. Nutr. 130, 2683-2690.

Horst, R.L., Goff, J.P., Reinhardt, T.A., 1997. Calcium and Vitamin D Metabolism During Lactation 1. J. Mammary Gland Biol. Neoplasia 2, 253-263.

Huffman, S.L., 1984. Determinants of breastfeeding in developing countries: overview and policy implications. Stud. Fam. Plann. 15, 170-83.

Humphrey, L.T., 2010. Weaning behaviour in human evolution. Semin. Cell Dev. Biol. 21, 45361. doi:10.1016/j.semcdb.2009.11.003

Hurtado, A.M., Hill, K., Hurtado, I., Kaplan, H., 1992. Trade-offs between female food acquisition and child care among Hiwi and Ache foragers. Hum. Nat. 3, 185-216. doi:10.1007/BF02692239

Kalanda, B.F., Verhoeff, F.H., Brabin, B.J., 2006. Breast and complementary feeding practices in relation to morbidity and growth in Malawian infants. Eur. J. Clin. Nutr. 60, 401-7. doi:10.1038/sj.ejcn.1602330

King, J., Ashworth, A., 1987. Historical review of the changing pattern of infant feeding in developing countries: the case of Malaysia, the Caribbean, Nigeria, and Zaire. Soc. Sci. Med. 25, 1307-1320.

Koplin, J.J., Osborne, N.J., Wake, M., Martin, P.E., Gurrin, L.C., Robinson, M.N., Tey, D., Slaa, M., Thiele, L., Miles, L., Anderson, D., Tan, T., Dang, T.D., Hill, D.J., Lowe, A.J., Matheson, M.C., Ponsonby, A.-L., Tang, M.L.K., Dharmage, S.C., Allen, K.J., 2010. Can early introduction of egg prevent egg allergy in infants? A population-based study. J. 
Allergy Clin. Immunol. 126, 807-13. doi:10.1016/j.jaci.2010.07.028

Kramer, M., Kakuma, R., 2002. The optimal duration of exclusive breastfeeding: a systematic review. Cochrane Libr. 1-47.

Levine, N.E., 1988. Women 's work and infant feeding : a case from rural Nepal. Ethnology 27, $231-251$.

Lutter, C.K., Morrow, A.L., 2013. Protection, promotion, and support and global trends in breastfeeding. Adv Nutr 4, 213-219. doi:10.3945/an.112.003111.Promotion

Martin, M., Blackwell, A.D., Gurven, M., Kaplan, H., 2013. Make new friends and keep the old? Parasite coinfection and comorbidity in Homo Sapiens, in: Brinkworth, J., Pechenkina, K. (Eds.), Primates, Pathogens, and Evolution. Springer Science + Buisniness Media, New York, pp. 363-387. doi:10.1007/978-1-4614-7181-3

Martin, M.A., 2015. Optimal exclusive breastfeeding duration: evidence of conflict and congruence in Tsimane mother-infant pairs. University of California Santa Barbara.

McAllister, L., Gurven, M., Kaplan, H., Stieglitz, J., 2012. Why do women have more children than they want? Understanding differences in women's ideal and actual family size in a natural fertility population. Am. J. Hum. Biol. 24, 786-99. doi:10.1002/ajhb.22316

McDade, T.W., Worthman, C.M., 1998. The weanling's dilemma reconsidered: a biocultural analysis of breastfeeding ecology. J Dev Behav Pediatr 19, 286-299.

McKenna, J.J., Gettler, L.T., 2016. There is no such thing as infant sleep, there is no such thing as breastfeeding, there is only breastsleeping. Acta Paediatr. Int. J. Paediatr. 105, 17-21. doi:10.1111/apa.13161

McNeilly, A.S., 1997. Lactation and fertility. J. Mammary Gland Biol. Neoplasia 2, 291-8. Meehan, C.L., Roulette, J.W., 2013. Early supplementary feeding among central African 
foragers and farmers: a biocultural approach. Soc. Sci. Med. 96, 112-20.

doi:10.1016/j.socscimed.2013.07.029

Meira-Machado, L., Cadarso-Suarez, C., Gude, F., Araujo, A., 2013. smoothHR: An R package for pointwise nonparametric estimation of hazard ratio curves of continuous predictors. Comput. Math. Methods Med. 2013.

Miner, E.J., Gurven, M., Kaplan, H., Gaulin, S.J.C., 2014. Sex difference in travel is concentrated in adolescence and tracks reproductive interests. Proc. R. Soc. Lond. B 281, 20141476.

Moffat, T., 2002. Breastfeeding, wage labor, and insufficient milk in peri-urban Kathmandu, Nepal. Med. Anthropol. 21, 165-188.

Moffat, T., 2001. A biocultural investigation of the weanling's dilemma in Kathmandu, Nepal: Do universal recommendations for weaning practices make sense? J Biosoc. Sci. 321-338. doi:10.1017/S0021932001003212

Nielsen, S.B., Reilly, J.J., Fewtrell, M.S., Eaton, S., Grinham, J., Wells, J.C.K., 2011. Adequacy of milk intake during exclusive breastfeeding: a longitudinal study. Pediatrics 128, e907-14. doi:10.1542/peds.2011-0914

Nommsen-Rivers, L. a, Dolan, L.M., Huang, B., 2012. Timing of stage II lactogenesis is predicted by antenatal metabolic health in a cohort of primiparas. Breastfeed. Med. 7, 43-9. doi:10.1089/bfm.2011.0007

Nordin, B.E.C., 1997. Calcium and osteoporosis. Nutrition 13, 664-686. doi:10.1016/S08999007(97)83011-0

Panter-Brick, C., 1993a. Seasonality of energy expenditure during pregnancy and lactation for rural Nepali women. Am. J. Clin. Nutr. 57, 620-628. 
Panter-Brick, C., 1993b. Mother-child food allocation and levels of subsistence activity in rural Nepal. Ecol. Food Nutr. 29, 319-333. doi:10.1080/03670244.1993.9991314

Panter-Brick, C., 1991. Lactation, birth spacing and maternal work-loads among two castes in rural Nepal. J. Biosoc. Sci. 23, 137-154.

Piperata, B. a, 2009. Variation in maternal strategies during lactation: the role of the biosocial context. Am. J. Hum. Biol. 21, 817-27. doi:10.1002/ajhb.20898

Powe, C.E., Knott, C.D., Conklin-brittain, N., 2010. Infant sex predicts breast milk energy content. Am. J. Hum. Biol. 22, 50-4. doi:10.1002/ajhb.20941

Prentice, A., Jarjou, L.M., Drury, P.J., Dewit, O., Crawford, M.A., 1989. Breast-milk fatty acids of rural Gambian mothers: effects of diet and maternal parity. J. Pediatr. Gastroenterol. Nutr. 8, 486-490. doi:10.1097/00005176-198905000-00011

Prescott, S.L., Smith, P., Tang, M., Palmer, D.J., Sinn, J., Huntley, S.J., Cormack, B., Heine, R.G., Gibson, R. a, Makrides, M., 2008. The importance of early complementary feeding in the development of oral tolerance: concerns and controversies. Pediatr. Allergy Immunol. 19, 375-80. doi:10.1111/j.1399-3038.2008.00718.x

Quinn, E.A., 2013. No evidence for sex biases in milk macronutrients, energy, or breastfeeding frequency in a sample of Filipino mothers. Am. J. Phys. Anthropol. 152, 209-16. doi:10.1002/ajpa.22346

Reilly, J.J., Wells, J.C.K., 2005. Duration of exclusive breast-feeding: introduction of complementary feeding may be necessary before 6 months of age. Br. J. Nutr. 94, 869. doi:10.1079/BJN20051601

Reyes-García, V., Paneque-Gálvez, J., Bottazzi, P., Luz, A.C., Gueze, M., Macía, M.J., OrtaMartínez, M., Pacheco, P., 2014. Indigenous land reconfiguration and fragmented 
institutions: A historical political ecology of Tsimane' lands (Bolivian Amazon). J. Rural Stud. 34, 282-291. doi:10.1016/j.jrurstud.2014.02.007

Sellen, D.W., 2009. Evolution of human lactation and complementary feeding: implications for understanding contemporary cross-cultural variation, in: Goldberg, G. (Ed.), BreastFeeding: Early Influences on Later Health. Springer Science + Buisniness Media B.V., pp. $253-282$.

Sellen, D.W., 2001. Comparison of infant feeding patterns reported for nonindustrial populations with current recommendations. J. Nutr. 131, 2707-2715.

Stieglitz, J., Beheim, B. a, Trumble, B.C., Madimenos, F.C., Kaplan, H., Gurven, M., 2015. Low mineral density of a weight-bearing bone among adult women in a high fertility population. Am. J. Phys. Anthropol. 156, 637-48. doi:10.1002/ajpa.22681

Strassmann, B.I., Gillespie, B., 2002. Life-history theory, fertility and reproductive success in humans. Proc. Biol. Sci. 269, 553-62. doi:10.1098/rspb.2001.1912

Tully, K.P., Ball, H.L., 2013. Trade-offs underlying maternal breastfeeding decisions: A conceptual model. Matern. Child Nutr. 9, 90-98. doi:10.1111/j.1740-8709.2011.00378.x

Valeggia, C., Ellison, P.T., 2009. Interactions between metabolic and reproductive functions in the resumption of postpartum fecundity. Am. J. Hum. Biol. 21, 559-66. doi:10.1002/ajhb.20907

Veile, A., Martin, M., McAllister, L., Gurven, M., 2014. Modernization is associated with intensive breastfeeding patterns in the Bolivian Amazon. Soc. Sci. Med. 100, 148-158. doi:10.1016/j.socscimed.2013.10.034

Victora, C.G., Bahl, R., Barros, A.J.D., França, G.V.A., Horton, S., Krasevec, J., Murch, S., Sankar, M.J., Walker, N., Rollins, N.C., 2016. Breastfeeding in the 21st century: 
epidemiology, mechanisms, and lifelong effect. Lancet 387, 475-490. doi:10.1016/S01406736(15)01024-7

Waterlow, J.C., 1981. Observations on the suckling's dilemma--a personal view. J. Hum. Nutr. $35,85-98$.

Wennergren, G., 2009. What if it is the other way around? Early introduction of peanut and fish seems to be better than avoidance. Acta Paediatr. 98, 1085-7. doi:10.1111/j.16512227.2009.01342.x

Wilson, W., Milner, J., Bulkan, J., Ehlers, P., 2006. Weaning practices of the Makushi of Guyana and their relationship to infant and child mortality: a preliminary assessment of international recommendations. Am J Hum Bio 18, 312-324. doi:10.1002/ajhb

Winking, J., Gurven, M., Kaplan, H., Stieglitz, J., 2009. The goals of direct paternal care among a South Amerindian population. Am. J. Phys. Anthropol. 139, 295-304. doi:10.1002/ajpa.20981

World Health Organization, 2007. Indicators for assessing infant and young child feeding practices. Young 2007, 1-19.

Wright, A.L., 2001. The Rise of Breastfeeding in the United States. Pediatr. Clin. North Am. 48, 1-12. doi:10.1016/S0031-3955(05)70282-0 


\section{Artwork and Tables with captions}

Table 1. Subject characteristics for sample and by study group.

\begin{tabular}{|c|c|c|c|c|c|c|}
\hline \multirow[t]{2}{*}{$\begin{array}{l}\text { Descriptor } \\
\text { (Range, mean } \pm \text { SD) }\end{array}$} & \multicolumn{2}{|c|}{$\begin{array}{l}\text { All Subjects } \\
\text { Surveyed }\end{array}$} & \multicolumn{2}{|c|}{$\begin{array}{l}\text { Initial Interview } \\
\text { Only }\end{array}$} & \multicolumn{2}{|c|}{$\begin{array}{l}\text { Prospective } \\
\text { Follow-Up }\end{array}$} \\
\hline & $\mathrm{N}$ & $\%$ & $\mathrm{~N}$ & $\%$ & $\mathrm{~N}$ & $\%$ \\
\hline Infant sex & \multicolumn{2}{|l|}{161} & \multicolumn{2}{|c|}{117} & \multicolumn{2}{|l|}{44} \\
\hline Male & 92 & 57.1 & 62 & 53.0 & 30 & 68.1 \\
\hline Female & 69 & 42.8 & 55 & 47.0 & 14 & 31.8 \\
\hline Infant age (mos.) & \multicolumn{2}{|c|}{$(0-35,13.8 \pm 9.8)$} & \multicolumn{2}{|c|}{$(0-35,17.4 \pm 9.0)$} & \multicolumn{2}{|c|}{$(0-10,4.3 \pm 10.5)$} \\
\hline $0-5$ & 46 & 28.5 & 17 & 14.5 & 29 & 65.9 \\
\hline $6-11$ & 27 & 16.8 & 12 & 10.3 & 15 & 34.1 \\
\hline $12-23$ & 61 & 37.9 & 61 & 52.1 & - & - \\
\hline $24-35$ & 27 & 16.8 & 27 & 23.1 & - & - \\
\hline Maternal age (yrs.) & \multicolumn{2}{|c|}{$(14-49,26.9 \pm 8.5)$} & \multicolumn{2}{|c|}{$(14-49,26.7 \pm 8.3)$} & \multicolumn{2}{|c|}{$(14-45,27.6 \pm 9.1)$} \\
\hline$<20$ & 33 & 20.4 & 24 & 20.5 & 9 & 20.5 \\
\hline 20 to 29 & 71 & 44.1 & 52 & 44.4 & 19 & 43.1 \\
\hline 30 to 39 & 38 & 23.6 & 29 & 24.8 & 9 & 20.5 \\
\hline 40 and over & 19 & 11.8 & 12 & 10.3 & 7 & 15.9 \\
\hline Number of births & \multicolumn{2}{|c|}{$(1-13,5 \pm 3)$} & \multicolumn{2}{|c|}{$(1-13,4 \pm 3)$} & \multicolumn{2}{|c|}{$(1-12,5 \pm 3)$} \\
\hline No previous birth & 27 & 16.8 & 20 & 17.1 & 7 & 15.9 \\
\hline 2 to 8 births & 115 & 71.4 & 82 & 70.1 & 33 & 75.0 \\
\hline 9 or more & 19 & 11.8 & 15 & 12.8 & 4 & 9.1 \\
\hline \multicolumn{7}{|l|}{ Spanish fluency } \\
\hline None & 72 & 45.9 & 50 & 44.2 & 22 & 50.0 \\
\hline Conversational & 72 & 45.9 & 52 & 46.0 & 20 & 45.5 \\
\hline Fluent & 13 & 8.2 & 11 & 9.9 & 2 & 4.5 \\
\hline \multicolumn{7}{|l|}{ Region } \\
\hline Near Town & 88 & 54.7 & 78 & 66.7 & 10 & 22.7 \\
\hline Remote & 73 & 45.3 & 39 & 33.3 & 34 & 77.2 \\
\hline
\end{tabular}


Table 2. Estimates of age-appropriate infant and young child feeding practices in Tsimane children aged 0-23 months. Sample sizes for each indicator reflect WHO-specified age groups (in parentheses)

\begin{tabular}{lccc}
\hline Indicator (WHO, 2007) & $\mathrm{n}$ & $\%$ & $95 \% \mathrm{CI}$ \\
\hline Initiation of breastfeeding within 1 hr (0 -23 months) & $70 / 131$ & $53 \%$ & $(44.5 ; 62.1)$ \\
Ever breastfed (0 - 23 months) & $133 / 133$ & $100 \%$ & $(96.5 ; 1.00)$ \\
EBF under 6 months (0 - 5 months) & $29 / 45$ & $64 \%$ & $(48.7 ; 77.7)$ \\
CF by 6- 8 months (6 - 8 months) & $13 / 13$ & $100 \%$ & $(71.7 ; 100.0)$ \\
Breastfeeding at 1 year (12 - 15 months) & $18 / 18$ & $100 \%$ & $(78.1 ; 100.0)$ \\
Breastfeeding at 2 years (19 - 23 months) & $13 / 15$ & $87 \%$ & $(58.4 ; 97.7)$ \\
Predominant breastfeeding (0 - 6 months) & $5 / 45$ & $11 \%$ & $(4.2 ; 24.9)$ \\
Bottle-feeding ( $0-23$ months) & $14 / 133$ & $11 \%$ & $(6.1 ; 17.3)$ \\
\hline
\end{tabular}


Figure 1. Prevalence by age group of premastication (solids) and oral transfer (liquids) reported in all 24-hour dietary recalls with non-EBF children $(n=236)$

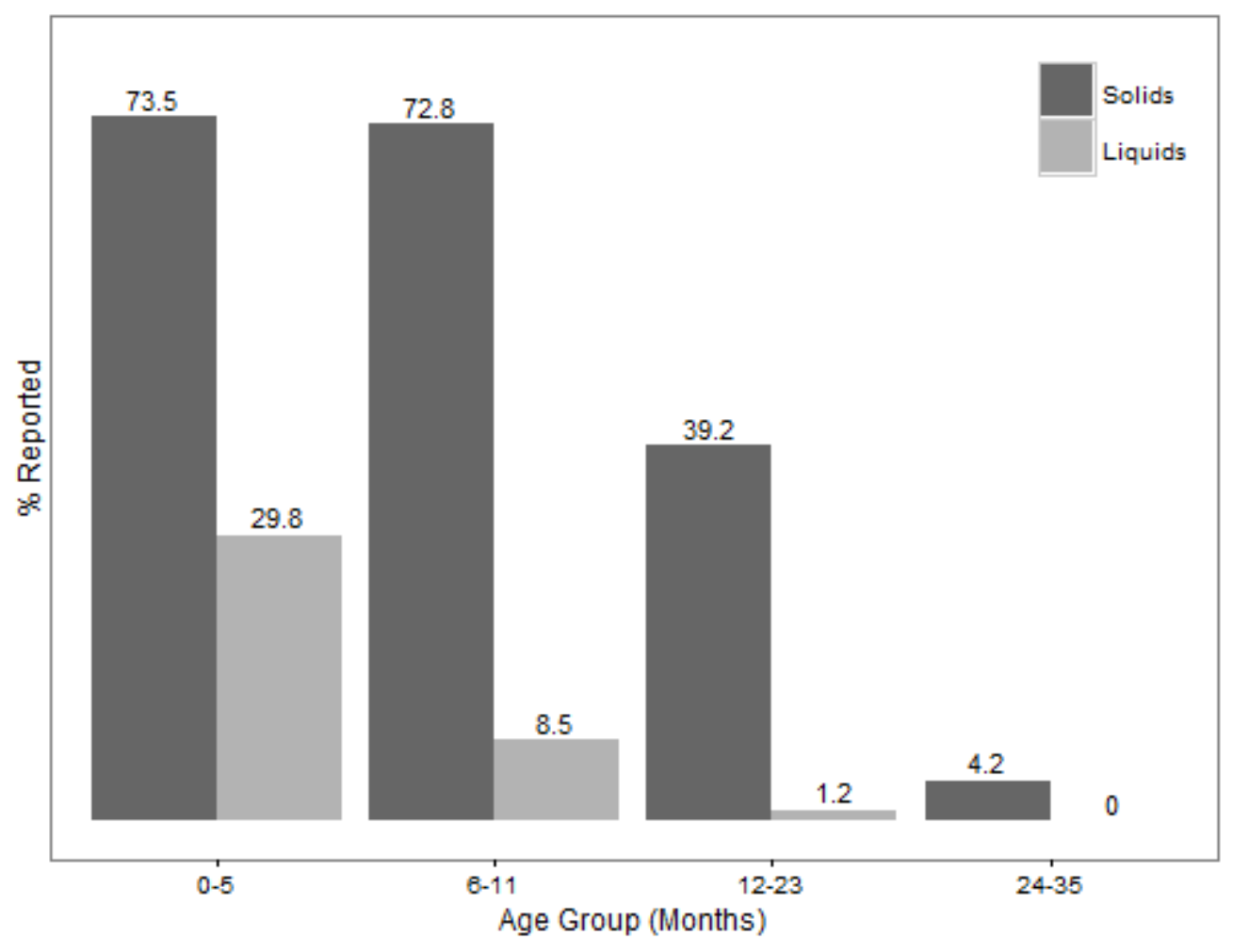


Figure 2. Tsimane mothers' self-reported reasons for introducing CF ( $n=137)$

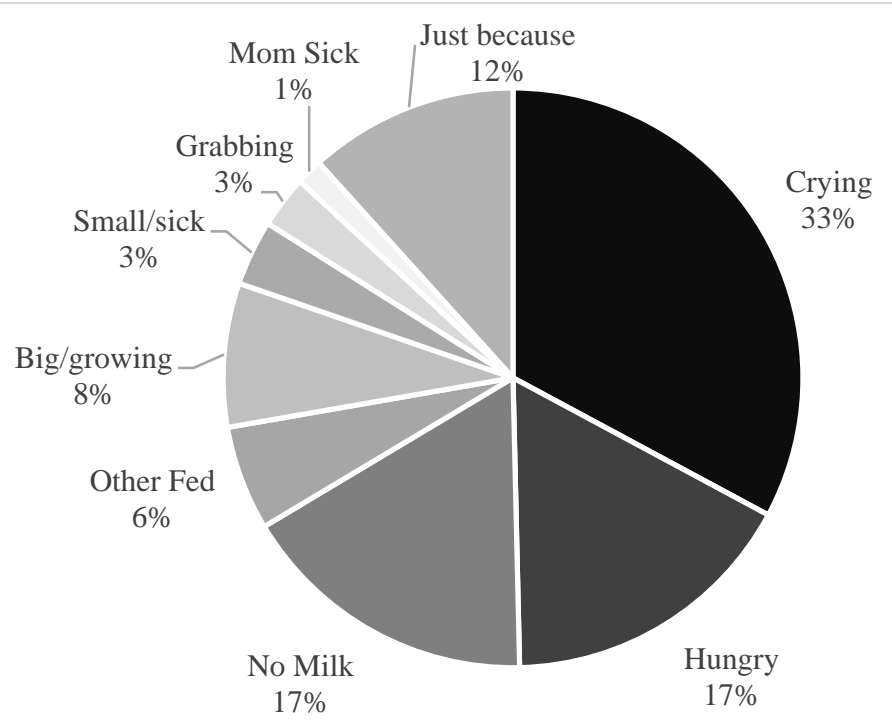


Table 3. Best fit Cox proportional hazards model of time to CF introduction (in days).

\begin{tabular}{lll}
\hline Factor & Hazard Ratio $(95 \% \mathrm{CI})$ & $p$-value \\
\hline Maternal height $(\mathrm{cm})$ & $0.96(0.92-0.99)$ & 0.024 \\
Parity & Nonlinear & 0.006 \\
Elapse time reported (days) & $1.00(1.00-1.00)$ & 0.220 \\
\hline
\end{tabular}


Figure 3. Log hazard ratios of CF by parity (risk is relative to minimum hazard at 5 births).

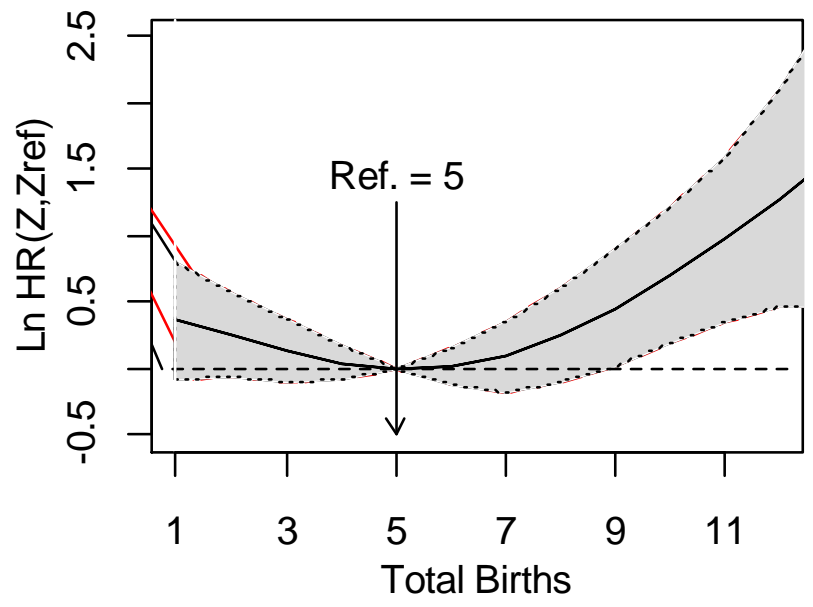


Table 4. Linear regression of age at $\mathrm{CF}$ introduction (months) in association with parity and perceived low milk supply (non-EBF infants, $\mathrm{n}=127$ ).

\begin{tabular}{llllr}
\hline & Model 1 & & Model 2 & \\
& Est. $(95 \% \mathrm{CI})$ & $p$ & Est. $(95 \% \mathrm{CI})$ & $p$ \\
\hline Low milk (yes) & -- & -- & $-3.43(-4.15 ;-2.72)$ & $<0.001$ \\
Parity group & & & & - \\
$\quad$ Prime $(2-8$ births $)$ & -- & -- & -- & 0.018 \\
$\quad$ Primiparous & $-0.65(-1.49 ; 0.20)$ & 0.135 & $-0.85(-1.56 ;-0.14)$ & 0.437 \\
$\quad$ High parity $(9+)$ & $-1.49(-2.49 ;-0.49)$ & 0.004 & $-0.32(-1.17 ; 0.51)$ & $<0.001$ \\
Maternal height $(\mathrm{cm})$ & $0.05(-1.51 ; 0.12)$ & 0.124 & $0.10(0.04 ; 0.15)$ & 0.486 \\
Days since CF intro & $0.0001(-0.0005 ; 0.002)$ & 0.214 & $0.0004(-0.001 ; 0.001)$ & \\
\hline
\end{tabular}


SSM-D-16-01320R1

Acknowledgements:

We would like to thank our host villages and families that participated in this study. Jaime Durbano, Bernabe Nate, Cody Elwell, and staff and researchers from the Tsimane Health and Life History Project provided invaluable assistance during field data collection. We also thank the three anonymous reviewers who provided helpful critiques on earlier versions of this manuscript. Funding for this research was provided by the Wenner-Gren Foundation and the National Science Foundation DDIG 1232370. Additional funding was provided by NIH/NIA Grant R01AG024119. 